coloproctology $2011 \cdot 33: 406$

DOI 10.1007/s00053-011-0236-6

๑) Springer-Verlag 2011

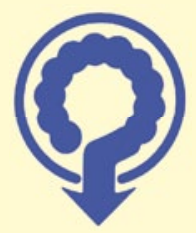

Schweizerische Arbeitsgruppe

für Koloproktologie (SAKP)

Mettlenweg 5

CH-8193 Eglisau

E-Mail: secretary@coloproct.ch

www.coloproct.ch

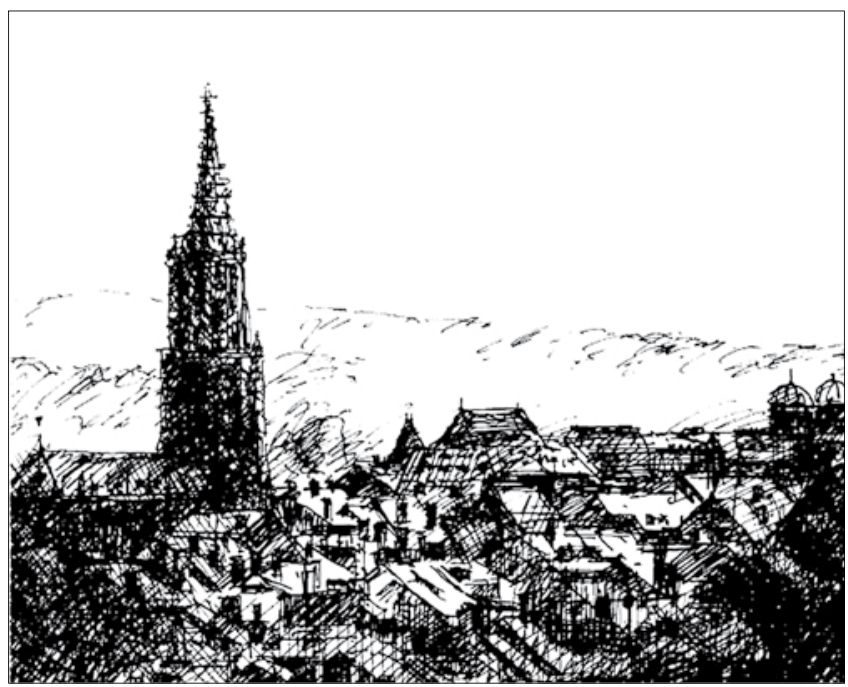

\title{
33. Schweizerische
}

Koloproktologie-Tagung

\section{Kolonkarzinom \& Beckenboden des}

\section{alten Menschen}

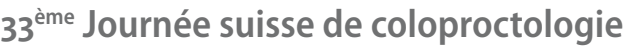 \\ Cancer colorectal \& plancher pelvien \\ de la personne agee}

Bern, 21.01.2012, 8.30 - 14.30 Uhr

\section{Kolonkarzinom/ Cancer colorectal}

Prävention - Screening / Prévention - Screening: Facts and Fantasy Prof. U. Marbet, Altdorf
Moderne histopathologische Diagnostik / Diagnostic histopathologique moderne Prof. W. Jochum, St. Gallen

Kontroversen der minimal invasiven Therapie /
Controverse de la chirurgie minimale invasive

Prof. J. Metzger, Luzern

Evidenz der modernen Chemotherapie? / Efficience de la chimiothérapie moderne?

Prof. M. Borner, Biel

Beckenboden des alten Menschen / Plancher pelvien de la personne âgée

Anti-Aging aus der Sicht des Urologen / Anti-aging du point de vue de l'urologue Prof. H.P. Gerbershagen, Ludwigsburg

Beckenboden- Deszensus: Gynäkologie / La descente périnéale: point de vue du gynécologue

Dr. D. Scheiner, Zürich

Beckenboden-Deszensus: Proktologie? / La descente périnéale: point de vue du proctologue Prof. F. Aigner, Innsbruck

\section{Workshops}

Analfissur in der Praxis / Les fissures anales au cabinet Dr. B. Roche, Genève

Der komplexe kolorektale Polyp / Les polypes colorectaux complexes

Prof. P. Bauerfeind, Zürich

Nachwuchsförderung in der

Koloproktologie / La relève en coloproctologie

Prof. F. Aigner, Innsbruck;

PD Dr. D. Dindo, Zürich;

PD Dr. F. Hetzer, Schaffhausen

\section{Akkreditierung / Accréditations}

SGG 6, SGIM 4.5, SGC/DDC 5

\section{Tagungssekretariat / Secrétariat du congrès}

Irene Moser, Mettlenweg 5,

8193 Eglisau

F 04486757 o1,

irenemoser@sunrise.ch

Tagungsort / Lieu du congrès Hotel Allegro,

Kornhausstrasse 3, 3000 Bern 25, www.kursaal-bern.ch 